\title{
Synthesis, characterization and antimicrobial analysis of Schiff bases of o-phenylenediamine and 2-aminopyridine-3-carboxylic acid with ofloxacin and their metal (II) complexes
}

\author{
Habila A.N. PUTAYA ${ }^{2 *}$, Naomi P. NDAHI ${ }^{1}$, Hauwa S. BELLO ${ }^{3}$, Grema MALA $^{1}$ \\ Adekunle A. OSUNLAJA ${ }^{1}$ and Hussaini GARBA ${ }^{2}$ \\ ${ }^{I}$ Chemistry Department, University of Maiduguri, Borno State-Nigeria. \\ ${ }^{2}$ Science Laboratory Technology, Ramat Polytechnic Maiduguri, Borno State-Nigeria. \\ ${ }^{3}$ Department of Microbiology, University of Maiduguri, Borno State-Nigeria. \\ *Corresponding author; E-mail: hputaya@yahoo.com, Tel: +2347035870121.
}

\section{ACKNOWLEDGEMENTS}

Sponsored By; Tertiary Education Trust Fund (TETFUND), Abuja-Nigeria and Ramat Polytechnic Maiduguri, Borno State-Nigeria.

\begin{abstract}
The increasing multi-drug resistance of microbes to the existing anti-biotic drugs has been of great concern to medical scientists and challenge has directly concerned pharmacologists, inorganic chemists, molecular microbiologists etc. This work attempted modifying a second generation fluoroquinolone (ofloxacin) toward attaining more improved activity. Schiff base Ligands $\mathrm{HL}^{1}$ and $\mathrm{HL}^{2}$ were obtained from separate condensation of o-phenylenediamine and 2-aminopyridine-3-carboxylic acid with ofloxacin in 2:1 and 1:1 mole ratio respectively. These ligands were complexed with chloride salts of $\mathrm{Ni}$ (II), $\mathrm{Mn}$ (II) and $\mathrm{Zn}$ (II) to afford their metal complexes. The new compounds were characterized on the basis of physicochemical properties, IR spectroscopy, UV-visible spectroscopy, Molar conductance and metal analysis. The IR spectra revealed that the metal ions coordinated with the ligands through azomethine nitrogen and carboxylato oxygen depicting tetradentate character with the formular type $\mathrm{ML}$ and $\mathrm{ML}_{2}$ for $\mathrm{HL}^{1}$ and $\mathrm{HL}^{2}$ metal complexes respectively. The antimicrobial evaluation of the ligands and their respective complexes in Staphylococcus aureus, Bacillus subtilis, Salmonella typhi, Escherichia coli, Aspergellus niger and Aspergellus fumigatus presented a promising to excellent activity except for A. niger which showed no activity in all the tested compounds.
\end{abstract}

() 2019 International Formulae Group. All rights reserved

Keywords: Schiff base, metal complexes, ofloxacin, o-phenylenediamine, 2-aminopyridine-3-carboxylic acid, antimicrobial activity.

\section{INTRODUCTION}

Schiff bases named after Hugo Schiff (1864), a German Scientist, are formed when primary amine reacts with ketones or aldehydes under specified mole ratio and conditions. These are usually compounds carrying imine or azomethine $(-\mathrm{C}=\mathrm{N}-)$ functional group, referred to as products of condensation of primary amines with carbonyl compounds (Ndahi and Nasiru, 2012). Schiff base forms an important class of the most widely used organic compounds and has a 
wide variety of applications in many fields including analytical, biological, medicinal, inorganic chemistry and as fine chemicals (Gangani and Parsania, 2014). The important and interesting roles of Schiff bases are as intermediates in the biologically important transmutation reactions. In azomethine derivatives, the $\mathrm{C}=\mathrm{N}$ linkage is essential for molecular interactions, as several azomethine have been reported to possess remarkable antibacterial, antifungal, anticancer and antimalarial activity (Sridhar et al., 2001; Raman et al., 2003). Heterocyclic Schiff bases have been reported as antibacterial and antifungal activities. Schiff base compounds have also been used as fine chemicals and medical substrates (Shrikrishna et al., 2012).

Quinolones are synthetic antibacterial compounds based on a 4-quinolone skeleton and are present in numerous natural products, especially in alkaloids. Many quinolones display interesting pharmacological activities and have found applications as pharmaceuticals, e.g antimalarial drugs, such as quinine or chloroquin (Jayashree et al., 2012). The present therapeutic arsenal notwithstanding, the efficacy of existing antimicrobial molecules is limited by the increasing multi-drug resistant microbes (Akpa et al., 2016) which is among the prime factors that ensures success of adaptive somatic modifications for enhanced living of these pathogens (Ngassam et al., 2017).

Metal complexed antibiotics have been reported to show either equal or increased antibacterial profile in comparison to the original drugs. Ofloxacin has been discreetly reported to possess the potential of interacting with the metal ions as a mono anionic bidentate ligand bound to the metal through the pyridone and carboxylate oxygen atoms having [M $\left.(\mathrm{Oflo})_{2}\left(\mathrm{H}_{2} \mathrm{O}\right)_{2}\right] \cdot \mathrm{nH}_{2} \mathrm{O}$ formula, where $\mathrm{M}=$ Metal ion (Sultana et al., 2013). There is a continuous and urgent need to discover new antimicrobial compounds with diverse chemical structures and novel mechanism of action because there have been an alarming increase in the incidence of new and re-emerging infectious disease (Anand et al., 2011).

However, extensive works have been reported on modifications of other fluoroquinolones especially ciprofloxacin, gatifloxacin, norfloxacin and levofloxacin both in Schiff base synthesis and their metal complexes (Sultana et al., 2013; Verma et al., 2013; Sadeek et al., 2014), but not much has been explored on ofloxacin toward enhancing its activity especially with respect to its imine Schiff bases and their metal complexes.

In view of the challenges of checking the menace of multi-drug resistant pathogens, this work was aimed at synthesizing some Schiff bases containing ofloxacin drug with ophenylenediamine and 2-aminopyridine-3carboxylic acid and complex them with $\mathrm{Ni}$ (II), Mn(II) and $\mathrm{Zn}(\mathrm{II}) \mathrm{Metal}$ ions. The expected newly synthesized ofloxacin derivatives were characterized using various methods and their antimicrobial potency evaluated with reference to the parent/referenced drugs.

\section{MATERIALS AND METHODS \\ Chemicals and Reagents}

All chemicals used in the synthesis were of analytical grade (AR). Ofloxacin antibiotic in pure (generic) form, ophenylenediamine and 2-aminopyridine-3carboxylic acid were all purchased from Sigma Aldrich through Bristol Scientific Company, Lagos, Nigeria. The metal (II) salts used were; $\mathrm{NiCl}_{2} \cdot 6 \mathrm{H}_{2} \mathrm{O}, \mathrm{MnCl}_{2} \cdot 4 \mathrm{H}_{2} \mathrm{O}$ and $\mathrm{Zn}$ $\left(\mathrm{NO}_{3}\right)_{2} \cdot 6 \mathrm{H}_{2} \mathrm{O}$. Solvents used for the synthesis and other probes were of absolute purity which include; distilled water, methanol, ethanol, chloroform, n-hexane, benzene and acetone. All new compounds were characterized on the basis of conductance measurement using DDS-307 Conductometer bridge. The melting point temperatures were determined using Gallenkamp melting apparatus and are uncorrected. The metal content of the complexes was determined using complexometric titration with EDTA. The electronic spectral data were recorded with UV-Vis spectrophotometer at $800-200$ 
$\mathrm{nm}$ and the FTIR analysis of the compounds was carried out in the range of $500-4000 \mathrm{~cm}^{-1}$ on SHIMADZU Corporation FTIR-8400S spectrophotometer. Disc diffusion method was used to evaluate the antimicrobial activities of the synthesized ligands and their metal (II) complexes against some strains of bacteria such as Staphylococcus aureus, Bacillus subtilis, Salmonella typhi and Escherichia coli and fungi like Aspergillus niger and Aspergillus fumigatus.

\section{Synthesis of the ligands (ofloxacin imines)}

The Schiff base ligands (ofloxacinimines) were synthesized using a literature procedure (Imran et al., 2007; Sadeek et al., 2014), by the condensation of ofloxacin antibiotic with respective substituted amines in the appropriate molar ratio in ethanol. Ofloxacin powder (2 mmol, $0.7227 \mathrm{~g}$ ) dissolved in $25 \mathrm{ml}$ methanol was mixed with o-phenylenediamine (1 mmol, $0.108 \mathrm{~g})$ dissolved in $12.5 \mathrm{ml}$ methanol, (2:1) in a round bottom flask. The mixture was subjected to reflux in the presence of 2 drops of glacial acetic acid for $3 \mathrm{~h}$. The resulting solution was concentrated on a water bath and allowed to cool at $0{ }^{0} \mathrm{C}$. The yellowish white solid formed $\left(\mathrm{HL}^{1}\right)$ was filtered, washed with ethanol and dried in a desiccator containing $\mathrm{CaCl}_{2}$. The same method was employed to prepare ofloxacin-2-aminopyridine-3carboxylic acid $\left(\mathrm{HL}^{2}\right)$ in 1:1 ratio combination and the whitish lemon yellow solid was obtained.

\section{Synthesis of metal complexes}

The prepared Schiff base ligands $(0.02$ mol) were dissolved in $25 \mathrm{ml}$ methanol and mixed with respective transition metal salts (0.01 mol) e.g $\mathrm{NiCl}_{2} \cdot 6 \mathrm{H}_{2} \mathrm{O}$ in $20 \mathrm{ml}$ methanol (1:1 or $1: 2$ ) ratio for $\mathrm{HL}^{1}$ and $\mathrm{HL}^{2}$ ligands respectively. The reaction mixture was refluxed for $3 \mathrm{~h}$, after which it was concentrated and cooled at room temperature which gave coloured precipitates. The precipitates were filtered, washed with methanol and dried in a desiccator containing $\mathrm{CaCl}_{2}$.

\section{Antimicrobial Assay}

Antimicrobial activity of the complexes, amines used and ligands were evaluated by Disc diffusion method as reported by Imran et al. (2007) and Al-Bayati et al. (2010) as adopted by Usman et al. (2007); Ndahi et al. (2012); Waziri et al. (2013) against different bacteria strains such as Staphylococcus aureus, Bacillus subtilis, Salmonella typhi and Escherichia coli, and also on some fungi strains like Aspergillus niger and Aspergillus fumigatus.

The nutrient agar medium (Peptone, Beef extract, $\mathrm{NaCl}$ and Agar-Agar) and $5 \mathrm{~mm}$ diameter paper disks (Whatman No. 1) were used. The investigated test compounds i.e. ligands, amines used and the complexes were dissolved $(30 \mu \mathrm{g}, 20 \mu \mathrm{g}, 10 \mu \mathrm{g})$ in methanol. $25 \mathrm{ml}$ nutrient agar media was poured into each Petri plates. After solidification, $0.1 \mathrm{ml}$ of test bacteria was spread over the medium. The disks of Whatman No.1 filter paper was placed at twelve equidistant places at a distance of $2 \mathrm{~cm}$ from the center in the inoculated petri plates. Another filter paper disks treated with methanol served as control whereas media with Ofloxacin $(30 \mu \mathrm{g} / \mathrm{ml})$ concentration (standard antibacterial) and Ketoconazole (30 $\mu \mathrm{g} / \mathrm{ml})$ concentration (standard antifungal) were used as positive controls or referenced drugs. The plates were then incubated for $24 \mathrm{~h}$ at $37{ }^{0} \mathrm{C}$ for bacteria and $48 \mathrm{~h}$ at room temperature for fungi, and the zone of inhibition around each disk were measured and recorded $(\mathrm{mm})$ as the degree of activity on the target microorganisms.

\section{Statistical analysis}

The raw data obtained from in vitro assay for minimum inhibition zone (mm) activity of the test agents on the selected panel of microorganism strains were subjected to one way ANOVA (Turkey-Kramer Multiple Comparisons Test) statistical analysis on pyramid Graphpad Instat, 2000 and expressed as \pm SEM. $P$ values $(\leq 0.05)$ were considered significant in this study to test the extent of potency of the new compounds among themselves across the columns as relative to the reference drugs. 


\section{RESULTS}

The analytical data of the Schiff base ligands $\left(\mathrm{HL}^{1} \& \mathrm{HL}^{2}\right)$ and their metal(II) complexes along with some physical properties are summarized in Table 1 . The presumed formula weights of the synthesized compounds were found to be; $794.90\left[\mathrm{HL}^{1}\right]$, $889.59 \quad\left[\mathrm{Ni}\left(\mathrm{HL}^{1}\right) .2 \mathrm{H}_{2} \mathrm{O}\right], \quad 885.84$ $\left[\mathrm{Mn}\left(\mathrm{HL}^{1}\right) \cdot 2 \mathrm{H}_{2} \mathrm{O}\right]$ and 896.28 $\left[\mathrm{Zn}\left(\mathrm{HL}^{1}\right) \cdot 2 \mathrm{H}_{2} \mathrm{O}\right]$. The common colours of the ofloxacin derivatives were yellow and green crystals with various intensity. The conductivity measurement $\left(\mathrm{Scm}^{2} \mathrm{~mol}^{-1}\right)$ were found as; $5.3 \times 10^{-5}\left[\mathrm{HL}^{1}\right], 5.2 \times 10^{-4}$ [ $\left.\mathrm{Ni}\left(\mathrm{HL}^{1}\right) \cdot 2 \mathrm{H}_{2} \mathrm{O}\right], 5.4 \times 10^{-4}\left[\mathrm{Mn}\left(\mathrm{HL}^{1}\right) \cdot 2 \mathrm{H}_{2} \mathrm{O}\right]$, and $4.0 \times 10^{-4}\left(\mathrm{Scm}^{2} \mathrm{~mol}^{-1}\right)$ for $\mathrm{Zn}\left(\mathrm{HL}^{1}\right) \cdot 2 \mathrm{H}_{2} \mathrm{O}$ which are relatively low values indicating possibly the compounds are weak or nonelectrolytes. Their melting points were determined as; $156-158{ }^{\circ} \mathrm{C}\left[\mathrm{HL}^{1}\right], 168-170{ }^{\circ} \mathrm{C}$ $\left[\mathrm{Ni}\left(\mathrm{HL}^{1}\right) \cdot 2 \mathrm{H}_{2} \mathrm{O}\right], \quad 162-164 \quad{ }^{\circ} \mathrm{C}$ $\left[\mathrm{Mn}\left(\mathrm{HL}^{1}\right) \cdot 2 \mathrm{H}_{2} \mathrm{O}\right]$ and $178-180 \quad{ }^{\circ} \mathrm{C}$ $\left[\mathrm{Zn}\left(\mathrm{HL}^{1}\right) \cdot 2 \mathrm{H}_{2} \mathrm{O}\right]$. The percentage yield was appreciable as it ranges from $61.18 \%$ $\left[\mathrm{Zn}\left(\mathrm{HL}^{1}\right) \cdot 2 \mathrm{H}_{2} \mathrm{O}\right]$ to $70.41 \%\left[\mathrm{Mn}\left(\mathrm{HL}^{1}\right) \cdot 2 \mathrm{H}_{2} \mathrm{O}\right]$. The metal analysis presented the percentage of metal in the complexes for both experimental (found) and theoretical (cal); $\begin{array}{lllll}6.60 \quad(6.85) & {\left[\mathrm{Ni}\left(\mathrm{HL}^{1}\right) .2 \mathrm{H}_{2} \mathrm{O}\right],} & 6.20 \quad(5.86)\end{array}$ $\left[\mathrm{Mn}\left(\mathrm{HL}^{1}\right) \cdot 2 \mathrm{H}_{2} \mathrm{O}\right]$ and 7.29 (6.97) \% for $\left[\mathrm{Zn}\left(\mathrm{HL}^{1}\right) \cdot 2 \mathrm{H}_{2} \mathrm{O}\right]$ which are both in close agreement.

Similarly, Table 2 shows the physical properties of $\mathrm{HL}^{2}$ ligand and its metal(II) complexes as follows; Formula weights were proposed as; $481.50 \quad\left[\mathrm{HL}^{2}\right], \quad 1057.69$ $\left[\mathrm{Ni}\left(\mathrm{HL}^{2}\right)_{2} .2 \mathrm{H}_{2} \mathrm{O}\right], 1053.94\left[\mathrm{Mn}\left(\mathrm{HL}^{2}\right)_{2} .2 \mathrm{H}_{2} \mathrm{O}\right]$ and $1064.38 \quad\left[\mathrm{Zn}\left(\mathrm{HL}^{2}\right)_{2} \cdot 2 \mathrm{H}_{2} \mathrm{O}\right]$ complex. Similar colours but with different intensities were observed for $\mathrm{HL}^{2}$ compounds which include pale yellow, whitish green, yellow and pale yellow for all the compounds respectively. Conductivity $\left(\mathrm{Scm}^{2} \mathrm{~mol}^{-1}\right) ; 6.1 \times$ $10^{-5}\left[\mathrm{HL}^{2}\right], 5.8 \times 10^{-4}\left[\mathrm{Ni}\left(\mathrm{HL}^{2}\right)_{2} .2 \mathrm{H}_{2} \mathrm{O}\right], 7 \times$ $10^{-4} \quad\left[\mathrm{Mn}\left(\mathrm{HL}^{2}\right)_{2} \cdot 2 \mathrm{H}_{2} \mathrm{O}\right]$ and $6 \times 10^{-5}$ $\left[\mathrm{Zn}\left(\mathrm{HL}^{2}\right)_{2} \cdot 2 \mathrm{H}_{2} \mathrm{O}\right]$. Melting points are presented as; $132-134{ }^{\circ} \mathrm{C}\left[\mathrm{HL}^{2}\right], 186-188{ }^{\circ} \mathrm{C}$ $\left[\mathrm{Ni}\left(\mathrm{HL}^{2}\right)_{2} .2 \mathrm{H}_{2} \mathrm{O}\right], \quad 185-187 \quad{ }^{\circ} \mathrm{C}$ $\left[\mathrm{Mn}\left(\mathrm{HL}^{2}\right)_{2} \cdot 2 \mathrm{H}_{2} \mathrm{O}\right]$ and $186-189 \quad{ }^{\circ} \mathrm{C}$ $\left[\mathrm{Zn}\left(\mathrm{HL}^{2}\right)_{2} \cdot 2 \mathrm{H}_{2} \mathrm{O}\right]$. Percentage (\%) yields; $70.41 \% \quad[\mathrm{HL} 2], \quad 69.60 \% \quad\left[\mathrm{Ni}\left(\mathrm{HL}^{2}\right)_{2} .2 \mathrm{H}_{2} \mathrm{O}\right]$,
$67.89 \%\left[\mathrm{Mn}\left(\mathrm{HL}^{2}\right)_{2} .2 \mathrm{H}_{2} \mathrm{O}\right]$ and $68.29 \%$ $\left[\mathrm{Zn}\left(\mathrm{HL}^{2}\right)_{2} \cdot 2 \mathrm{H}_{2} \mathrm{O}\right]$. The percentage (\%) metal content of the complexes were found to be; $5.55(5.28) \%\left[\mathrm{Ni}\left(\mathrm{HL}^{2}\right)_{2} .2 \mathrm{H}_{2} \mathrm{O}\right.$ ], $5.21(5.49) \%$ $\left[\mathrm{Mn}\left(\mathrm{HL}^{2}\right)_{2} .2 \mathrm{H}_{2} \mathrm{O}\right]$ and $6.14 \quad(6.54) \%$ $\left[\mathrm{Zn}\left(\mathrm{HL}^{2}\right)_{2} \cdot 2 \mathrm{H}_{2} \mathrm{O}\right]$. Table 3 presented relevant infrared (IR) spectra of the parent drug (ofloxacin), amine used, synthesized ligands and their metal (II) complexes $\left(\mathrm{cm}^{-1}\right)$. The important spectra for the drug (ofloxacin) as obtained in Table 3 include; $\mathrm{v}\left(\mathrm{NH}_{2}\right)-2924$ $\mathrm{cm}^{-1}, \mathrm{v}(\mathrm{OH})-3803 \mathrm{~cm}^{-1}, 3749 \mathrm{~cm}^{-1}, \mathrm{v}(\mathrm{C}=\mathrm{N})-$ $1519 \mathrm{~cm}^{-1}$ and $\mathrm{v}(\mathrm{C}-\mathrm{N})-1458 \mathrm{~cm}^{-1}$. The amine (o-phenylenediamine) showed the following spectra; $\mathrm{v}\left(\mathrm{NH}_{2}\right)-3363 \mathrm{~cm}^{-1}, 2924 \mathrm{~cm}^{-1}$, $\mathrm{v}(\mathrm{OH})-3865 \mathrm{~cm}^{-1}, \mathrm{v}(\mathrm{C}=\mathrm{N})-1627 \mathrm{~cm}^{-1}, 1535$ $\mathrm{cm}^{-1}$ and $\mathrm{v}(\mathrm{C}-\mathrm{N})-1496 \mathrm{~cm}^{-1}$. 2aminopyridene-3-carboxylic acid had the following guide spectra; $\mathrm{v}\left(\mathrm{NH}_{2}\right)-3255 \mathrm{~cm}^{-1}$, $\mathrm{v}(\mathrm{OH})-3865 \mathrm{~cm}^{-1}, 3749 \mathrm{~cm}^{-1}, \mathrm{v}(\mathrm{C}=\mathrm{N})-1697$ $\mathrm{cm}^{-1}, 1627 \mathrm{~cm}^{-1}$ and $\mathrm{v}(\mathrm{C}-\mathrm{N})-1458 \mathrm{~cm}^{-1} . \mathrm{HL}^{1}$ ligand spectra; $\mathrm{v}(\mathrm{OH})-3406 \mathrm{~cm}^{-1}, 3043 \mathrm{~cm}^{-1}$ $\mathrm{v}(\mathrm{C}=\mathrm{N})-1620 \mathrm{~cm}^{-1}, 1535 \mathrm{~cm}^{-1}, \mathrm{v}(\mathrm{H} 2 \mathrm{O})-995$ $\mathrm{cm}^{-1}, 960 \mathrm{~cm}^{-1}$ and $\mathrm{v}(\mathrm{C}-\mathrm{N})-1462 \mathrm{~cm}^{-1} . \mathrm{HL}^{2}$ ligand showed the same spectra as; $\mathrm{v}(\mathrm{OH})$ $3402 \mathrm{~cm}^{-1}, 3263 \mathrm{~cm}-1, \mathrm{v}(\mathrm{C}=\mathrm{N})-1631 \mathrm{~cm}^{-1}$, $1535 \mathrm{~cm}^{-1}, \mathrm{v}\left(\mathrm{H}_{2} \mathrm{O}\right)-964 \mathrm{~cm}^{-1}, 868 \mathrm{~cm}^{-1}$ and $\mathrm{v}(\mathrm{C}-\mathrm{N})-1465 \mathrm{~cm}^{-1}$. The metal complexes of the ligand presented more spectra such as; $\mathrm{Ni}\left(\mathrm{HL}^{1}\right) \cdot 2 \mathrm{H}_{2} \mathrm{O}$ showed absorption at $3406 \mathrm{~cm}^{-}$ ${ }_{1}^{1}$ assigned for $\mathrm{v}(\mathrm{OH}), 1643 \mathrm{~cm}^{-1}, 1535 \mathrm{~cm}^{-1}$ $\mathrm{v}(\mathrm{C}=\mathrm{N}), \quad 987 \mathrm{~cm}^{-1}$ attributed to $\mathrm{v}\left(\mathrm{H}_{2} \mathrm{O}\right)$ products vibration, $1489 \mathrm{~cm}^{-1}$ assigned to $\mathrm{v}(\mathrm{C}-\mathrm{N}), 516 \mathrm{~cm}^{-1}$ due to $\mathrm{v}(\mathrm{M}-\mathrm{N})$ and $615 \mathrm{~cm}^{-1}$ which is a non-ligand spectra of $\mathrm{v}(\mathrm{M}-\mathrm{O})$. $\mathrm{Mn}\left(\mathrm{HL}^{1}\right) \cdot 2 \mathrm{H}_{2} \mathrm{O}$ presented; $3398 \mathrm{~cm}^{-1} \mathrm{v}(\mathrm{OH})$, $1631 \mathrm{~cm}^{-1}, 1550 \mathrm{~cm}^{-1}$ for $\mathrm{v}(\mathrm{C}=\mathrm{N}), 983 \mathrm{~cm}^{-1}$ $\mathrm{v}\left(\mathrm{H}_{2} \mathrm{O}\right)$ vibration, $1473 \mathrm{~cm}^{-1}$ assigned to $\mathrm{v}(\mathrm{C}-$ $\mathrm{N}), 586 \mathrm{~cm}^{-1}$ for $\mathrm{v}(\mathrm{M}-\mathrm{N})$ and $451 \mathrm{~cm}^{-1}$ attributed to $\mathrm{v}(\mathrm{M}-\mathrm{O}) . \mathrm{Zn}\left(\mathrm{HL}^{1}\right) \cdot 2 \mathrm{H}_{2} \mathrm{O}$ showed these spectra as follows; $3402 \mathrm{~cm}^{-1} \mathrm{v}(\mathrm{OH})$, $1635 \mathrm{~cm}^{-1}, 1535 \mathrm{~cm}^{-1} \mathrm{v}(\mathrm{C}=\mathrm{N}), 960 \mathrm{~cm}^{-1}$ for $\mathrm{v}\left(\mathrm{H}_{2} \mathrm{O}\right), 1465 \mathrm{~cm}^{-1}$ assigned to $\mathrm{v}(\mathrm{C}-\mathrm{N}), 682$ $\mathrm{cm}^{-1}$ due to $\mathrm{v}(\mathrm{M}-\mathrm{N})$ and $405 \mathrm{~cm}^{-1}$ for $\mathrm{v}(\mathrm{M}-\mathrm{O})$. $\mathrm{Ni}\left(\mathrm{HL}^{2}\right)_{2} \cdot 2 \mathrm{H}_{2} \mathrm{O}$ complexes showed peaks at; $3363 \mathrm{~cm}^{-1}, 3255 \mathrm{~cm}^{-1}$ assigned to $\mathrm{v}(\mathrm{OH})$ vibration, $1639 \mathrm{~cm}^{-1}, 1546 \mathrm{~cm}^{-1}$ for $\mathrm{v}(\mathrm{C}=\mathrm{N})$, $879 \mathrm{~cm}^{-1}, 790 \mathrm{~cm}^{-1}$ due to $\mathrm{v}\left(\mathrm{H}_{2} \mathrm{O}\right), 1419 \mathrm{~cm}^{-1}$ $\mathrm{v}(\mathrm{C}-\mathrm{N}), 659 \mathrm{~cm}^{-1}, 578 \mathrm{~cm}^{-1}$ for $\mathrm{v}(\mathrm{M}-\mathrm{N})$ and $405 \mathrm{~cm}^{-1}$ attributed to $\mathrm{v}(\mathrm{M}-\mathrm{O})$. $\mathrm{Mn}\left(\mathrm{HL}^{2}\right)_{2} \cdot 2 \mathrm{H}_{2} \mathrm{O}$ presented these vibration 
spectra at; $3402 \mathrm{~cm}^{-1}, 3255 \mathrm{~cm}^{-1}$ assigned to $\mathrm{v}(\mathrm{OH}), 1543 \mathrm{~cm}^{-1}, 1639 \mathrm{~cm}^{-1}$ due to $\mathrm{v}(\mathrm{C}=\mathrm{N})$, $875 \mathrm{~cm}^{-1}, 790 \mathrm{~cm}^{-1}$ for $\mathrm{v}(\mathrm{OH}), 1481 \mathrm{~cm}^{-1} \mathrm{v}(\mathrm{C}-$ $\mathrm{N}), 663 \mathrm{~cm}^{-1}, 582 \mathrm{~cm}^{-1}$ for $\mathrm{v}(\mathrm{M}-\mathrm{N})$ and 478 $\mathrm{cm}^{-1} \quad \mathrm{v}(\mathrm{M}-\mathrm{O}) . \quad \mathrm{Zn}\left(\mathrm{HL}^{2}\right)_{2} \cdot 2 \mathrm{H}_{2} \mathrm{O}$ had its absorption spectra at; $3398 \mathrm{~cm}^{-1}$ for $\mathrm{v}(\mathrm{OH})$, $1693 \mathrm{~cm}^{-1}, 1635 \mathrm{~cm}^{-1}$ assigned to $\mathrm{v}(\mathrm{C}=\mathrm{N}), 864$ $\mathrm{cm}^{-1}, 779 \mathrm{~cm}^{-1}$ due to $\mathrm{v}\left(\mathrm{H}_{2} \mathrm{O}\right), 1462 \mathrm{~cm}^{-1}$ for $\mathrm{v}(\mathrm{C}-\mathrm{N}), 644 \mathrm{~cm}^{-1}, 597 \mathrm{~cm}^{-1}$ for $\mathrm{v}(\mathrm{M}-\mathrm{N})$ and $405 \mathrm{~cm}^{-1}$ due to non-ligand $\mathrm{v}(\mathrm{M}-\mathrm{O})$ vibration. Table 4 showed electronic absorption spectra of the ligands and their metal complexes; The $\mathrm{HL}^{1}$ - (oflox-o-phdn) showed electronic spectra at $K_{\max } 308 \mathrm{~nm}$ or $32468 \mathrm{~cm}^{-1}$ wave number assigned to $\mathrm{n} \rightarrow \pi^{*}$. $\mathrm{HL}^{2}$ - (oflox-2Apydn) presented its spectra at $\Lambda_{\max } 362 \mathrm{~nm}$ or $27624 \mathrm{~cm}^{-1}$ wave number also assigned to $\mathrm{n} \rightarrow \pi^{*}$ transition. $\mathrm{Ni}\left(\mathrm{HL}^{1}\right) \cdot 2 \mathrm{H}_{2} \mathrm{O}$ showed the electronic spectra at $K_{\max } 295 \mathrm{~nm}$ or $33898 \mathrm{~cm}^{-}$ ${ }^{1}$ wave number which is suggested for $n \rightarrow \pi^{*}$ transition. The electronic spectra for $\mathrm{Mn}$ $\left(\mathrm{HL}^{1}\right) .2 \mathrm{H}_{2} \mathrm{O}$ were observed at $K_{\max } 343 \mathrm{~nm}$ or $29155 \mathrm{~cm}^{-1}$ assumed to be for metal to ligand charge transfer (MLCT). $\mathrm{Zn} \quad\left(\mathrm{HL}^{1}\right) \cdot 2 \mathrm{H}_{2} \mathrm{O}$ electronic spectra was observed at $\Lambda_{\max } 349$ $\mathrm{nm}$ or $28653 \mathrm{~cm}^{-1}$ wave number also attributed to MLCT.

The $\mathrm{HL}^{2}$ complexes also showed irregular absorption spectra for $\mathrm{Ni}\left(\mathrm{HL}^{2}\right)_{2} \cdot 2 \mathrm{H}_{2} \mathrm{O}$ at $K_{\max } 238 \mathrm{~nm}$ or $42017 \mathrm{~cm}^{-1}$ assigned to $\pi \rightarrow \pi^{*}$ transition. $\mathrm{Mn}\left(\mathrm{HL}^{2}\right)_{2} \cdot 2 \mathrm{H}_{2} \mathrm{O}$ showed its absorption at $K_{\max } 221 \mathrm{~nm}$ or 45249 $\mathrm{cm}^{-1}$ attributed to $\pi \rightarrow \pi^{*}$ electronic transition. $\mathrm{Zn}\left(\mathrm{HL}^{2}\right)_{2} \cdot 2 \mathrm{H}_{2} \mathrm{O}$ had its absorption spectra at $\kappa_{\max } 353 \mathrm{~nm}$ or $28329 \mathrm{~cm}^{-1}$ assumed to be due to MLCT.

Tables 5 and 6 presented results for the in vitro antimicrobial activity evaluation for the Schiff base ligands and their metal (II) complexes. The synthesized compounds were tested against four medically important bacterial strains such as Staphylococcus aureus, Bacillus subtilis, Salmonella typhi and Escherichia coli and, two fungal strains Aspergellus niger and Aspergellus fumigatus. The results of the activity of the ligands and their complexes on the bacterial strains are presented in millimeter zone of inhibition at three serial dilutions of $30 \mu \mathrm{g} / \mathrm{ml}, 20 \mu \mathrm{g} / \mathrm{ml} \&$ $10 \mu \mathrm{g} / \mathrm{ml}$. The observed results showed promising to excellent activity of the novel compounds on the test microbes (Table 5) while the fungal strains resisted all the compounds of $\mathrm{HL}^{2}$ ligands as shown in Table 6.

The statistical analysis results are also presented in Tables 5 and 6 as superscripts along columns. Different superscripts along the same column are significantly $(\mathrm{P}<0.05)$ different and are referenced to the parent/reference drugs at the highest concentration of $30 \mu \mathrm{g} / \mathrm{ml}$.

Table 1: Physical Characteristics of $\mathrm{HL}^{1}$ Ligand and its Metal (II) Complexes.

\begin{tabular}{lllllll}
\hline $\begin{array}{l}\text { Compound/Proposed } \\
\text { Formula }\end{array}$ & $\begin{array}{l}\text { F. } \\
\text { Weight }\end{array}$ & Colour & $\begin{array}{l}\text { Conductivity } \\
\left(\mathbf{S c m}^{2} \mathbf{m o l}^{-1}\right)\end{array}$ & $\begin{array}{l}\text { M.P } \\
\left({ }^{\mathbf{C}} \mathbf{C}\right)\end{array}$ & $\begin{array}{l}\text { Yield } \\
(\%)\end{array}$ & $\begin{array}{l}\text { \%M } \\
\text { Found(Cal) }\end{array}$ \\
\hline$\left(\mathrm{HL}^{1}\right) / \mathrm{C}_{42} \mathrm{H}_{44} \mathrm{~F}_{2} \mathrm{~N}_{8} \mathrm{O}_{6}$ & 794.90 & $\begin{array}{l}\text { Yellowish } \\
\text { white }\end{array}$ & $5.3 \times 10^{-5}$ & $156-158$ & 66.44 & - \\
$\mathrm{Ni}\left(\mathrm{HL}^{1}\right) .2 \mathrm{H}_{2} \mathrm{O}$ & 889.59 & Green & $5.2 \times 10^{-4}$ & $168-170$ & 66.44 & $6.60(6.85)$ \\
$\mathrm{Mn}\left(\mathrm{HL}^{1}\right) .2 \mathrm{H}_{2} \mathrm{O}$ & 885.838 & Yellow & $5.4 \times 10^{-4}$ & $162-164$ & 70.41 & $6.20(5.86)$ \\
$\mathrm{Zn}\left(\mathrm{HL}^{1}\right) .2 \mathrm{H}_{2} \mathrm{O}$ & 896.28 & $\begin{array}{l}\text { Lemon } \\
\text { Yellow }\end{array}$ & $4.0 \times 10^{-4}$ & $178-180$ & 61.18 & $7.29(6.97)$ \\
& & & & & \\
\hline
\end{tabular}


Table 2: Physical Characteristics of $\mathrm{HL}^{2}$ Ligand and its Metal (II) Complexes.

\begin{tabular}{|c|c|c|c|c|c|c|}
\hline $\begin{array}{c}\text { Proposed } \\
\text { Formula }\end{array}$ & $\begin{array}{c}\text { F. } \\
\text { Weight }\end{array}$ & Colour & $\begin{array}{l}\text { Conductivity } \\
\left(\mathrm{Scm}^{2} \mathrm{~mol}^{-2}\right)\end{array}$ & $\begin{array}{l}\text { M.P } \\
\left({ }^{0} \mathrm{C}\right)\end{array}$ & $\begin{array}{c}\text { Yield } \\
(\%)\end{array}$ & $\begin{array}{c}\% \mathrm{M} \\
\text { Found(Cal) }\end{array}$ \\
\hline$\left(\mathrm{HL}^{2}\right) / \mathrm{C}_{24} \mathrm{H}_{24} \mathrm{FN}_{5} \mathrm{O}_{5}$ & 481.50 & Pale yellow & $6.1 \times 10^{-5}$ & $132-134$ & 70.41 & - \\
\hline $\mathrm{Ni}\left(\mathrm{HL}^{2}\right)_{2} \cdot 2 \mathrm{H}_{2} \mathrm{O}$ & 1057.69 & $\begin{array}{l}\text { Whitish } \\
\text { Green }\end{array}$ & $5.8 \times 10^{-4}$ & $186-188$ & 69.60 & $5.55(5.28)$ \\
\hline $\mathrm{Mn}\left(\mathrm{HL}^{2}\right)_{2} \cdot 2 \mathrm{H}_{2} \mathrm{O}$ & 1053.938 & Yellow & $7 \times 10^{-4}$ & $185-187$ & 67.85 & $5.21(5.49)$ \\
\hline $\mathrm{Zn}\left(\mathrm{HL}^{2}\right)_{2} \cdot 2 \mathrm{H}_{2} \mathrm{O}$ & 1064.38 & Pale Yellow & $6 \times 10^{-5}$ & $186-189$ & 68.29 & $6.14(6.54)$ \\
\hline
\end{tabular}

Table 3: Relevant Infrared Spectra of the Drug, Amines, Ligands and their Metal (II) Complexes $\left(\mathrm{cm}^{-1}\right)$.

\begin{tabular}{|c|c|c|c|c|c|c|c|}
\hline Compds. & $\mathbf{v}\left(\mathrm{NH}_{2}\right)$ & $\mathbf{v}(\mathbf{O H})$ & $\mathbf{v}(\mathrm{C}=\mathrm{N})$ & $\mathbf{v}\left(\mathrm{H}_{2} \mathbf{O}\right)$ & $\mathrm{v}(\mathrm{C}-\mathrm{N})$ & $\mathbf{v}(\mathbf{M}-\mathbf{N})$ & $\mathrm{v}(\mathrm{M}-\mathrm{O})$ \\
\hline Oflox. & 2924 sh & $\begin{array}{l}3803 w \\
3749 w\end{array}$ & $1519 w$ & - & $1458 \mathrm{~s}$ & - & - \\
\hline o-phdn & $\begin{array}{l}3363 w \\
2924 m\end{array}$ & $3865 w$ & $\begin{array}{l}1627 \mathrm{sh} \\
1535 \mathrm{~m}\end{array}$ & - & $1496 \mathrm{~s}$ & - & - \\
\hline 2-Apdn & $3255 \mathrm{sh}$ & $\begin{array}{l}3865 \mathrm{sh} \\
3749 \mathrm{w}\end{array}$ & $\begin{array}{l}1697 \mathrm{sh} \\
1627 \mathrm{~m}\end{array}$ & - & $1458 \mathrm{sh}$ & - & - \\
\hline $\mathrm{HL}^{1}$ & - & $\begin{array}{l}3406 \mathrm{sh} \\
3043 \mathrm{w}\end{array}$ & $\begin{array}{l}1620 \mathrm{~b} \\
1535 \mathrm{~m}\end{array}$ & $\begin{array}{l}995 w \\
960 m\end{array}$ & $1462 \mathrm{sh}$ & - & - \\
\hline $\mathrm{HL}^{2}$ & - & $\begin{array}{c}3402 \mathrm{~b} \\
3263 \mathrm{~m}\end{array}$ & $\begin{array}{l}1631 \mathrm{sh} \\
1535 \mathrm{~m}\end{array}$ & $\begin{array}{l}964 w \\
868 w\end{array}$ & $1465 \mathrm{~m}$ & - & - \\
\hline $\mathrm{Ni}\left(\mathrm{HL}^{1}\right) \cdot 2 \mathrm{H}_{2} \mathrm{O}$ & - & $3406 s$ & $\begin{array}{l}1643 \mathrm{sh} \\
1535 \mathrm{~m}\end{array}$ & $987 w$ & $1489 w$ & $516 \mathrm{w}$ & $613 w$ \\
\hline $\mathrm{Mn}\left(\mathrm{HL}^{1}\right) \cdot 2 \mathrm{H}_{2} \mathrm{O}$ & - & 3398sh & $\begin{array}{c}1631 \mathrm{~b} \\
1550 \mathrm{~m}\end{array}$ & $983 \mathrm{~m}$ & $1473 \mathrm{~m}$ & $586 w$ & $451 \mathrm{w}$ \\
\hline $\mathrm{Zn}\left(\mathrm{HL}^{1}\right) \cdot 2 \mathrm{H}_{2} \mathrm{O}$ & - & $3402 \mathrm{sh}$ & $\begin{array}{l}1635 \mathrm{~m} \\
1535 \mathrm{~m}\end{array}$ & $960 w$ & $1465 \mathrm{~m}$ & $682 \mathrm{w}$ & $405 \mathrm{~m}$ \\
\hline $\mathrm{Ni}\left(\mathrm{HL}^{2}\right)_{2} \cdot 2 \mathrm{H}_{2} \mathrm{O}$ & - & $\begin{array}{l}3363 w \\
3255 \mathrm{sh}\end{array}$ & $\begin{array}{l}1639 \mathrm{~m} \\
1546 \mathrm{~m}\end{array}$ & $\begin{array}{c}879 w \\
790 s\end{array}$ & $1419 \mathrm{~m}$ & $\begin{array}{l}659 \mathrm{~s} \\
578 \mathrm{~b}\end{array}$ & $405 \mathrm{sh}$ \\
\hline $\mathrm{Mn}\left(\mathrm{HL}^{2}\right)_{2} \cdot 2 \mathrm{H}_{2} \mathrm{O}$ & - & $\begin{array}{l}3402 \mathrm{sh} \\
3255 \mathrm{w}\end{array}$ & $\begin{array}{l}1543 \mathrm{~m} \\
1639 \mathrm{~m}\end{array}$ & $\begin{array}{l}875 \mathrm{w} \\
790 \mathrm{~m}\end{array}$ & $1481 w$ & $\begin{array}{c}663 \mathrm{sh} \\
582 \mathrm{~b}\end{array}$ & $478 w$ \\
\hline $\mathrm{Zn}\left(\mathrm{HL}^{2}\right)_{2} \cdot 2 \mathrm{H}_{2} \mathrm{O}$ & - & 3398sh & $\begin{array}{l}1693 w \\
1635 m\end{array}$ & $\begin{array}{l}864 w \\
779 m\end{array}$ & $1462 w$ & $\begin{array}{l}644 \mathrm{w} \\
597 \mathrm{w}\end{array}$ & $405 \mathrm{sh}$ \\
\hline
\end{tabular}

Key: oflox=ofloxacin, o-phdn=o-phenylenediamine, 2-Apdn=2-aminopyridine-3-carboxylic acid, HL=ligand, sh- sharp, $\mathrm{w}=$ weak, $\mathrm{m}=$ medium, $\mathrm{s}=$ strong, $\mathrm{b}=$ broad 
H. A.N. PUTAYA et al. / Int. J. Biol. Chem. Sci. 14(1): 263-278, 2020

Table 4: Electronic Absorption Spectra of the Ligands and their Metal Complexes.

\begin{tabular}{cccc}
\hline Compds. & $\lambda_{\text {max }}(\mathbf{n m})$ & Wave number $\left(\mathbf{c m}^{-1}\right)$ & Assignment \\
\hline $\mathrm{HL}^{1}($ oflox-ophdn $)$ & 308 & 32468 & $\mathrm{n} \rightarrow \pi^{*}$ \\
$\mathrm{HL}^{2}$ (oflox-2-Apydn) & 362 & 27624 & $\mathrm{n} \rightarrow \pi^{*}$ \\
$\mathrm{Ni}\left(\mathrm{HL}^{1}\right) \cdot 2 \mathrm{H}_{2} \mathrm{O}$ & 295 & 33898 & $\mathrm{n} \rightarrow \pi^{*}$ \\
$\mathrm{Mn}\left(\mathrm{HL}^{1}\right) \cdot 2 \mathrm{H}_{2} \mathrm{O}$ & 343 & 29155 & $\mathrm{MLCT}$ \\
$\mathrm{Zn}\left(\mathrm{HL}^{1}\right) \cdot 2 \mathrm{H}_{2} \mathrm{O}$ & 349 & 28653 & $\mathrm{MLCT}$ \\
$\mathrm{Ni}\left(\mathrm{HL}^{2}\right)_{2} \cdot 2 \mathrm{H}_{2} \mathrm{O}$ & 238 & 42017 & $\pi \rightarrow \pi^{*}$ \\
$\mathrm{Mn}\left(\mathrm{HL}^{2}\right)_{2} \cdot 2 \mathrm{H}_{2} \mathrm{O}$ & 221 & 45249 & $\pi \rightarrow \pi^{*}$ \\
$\mathrm{Zn}\left(\mathrm{HL}^{2}\right)_{2} \cdot 2 \mathrm{H}_{2} \mathrm{O}$ & 353 & 28329 & MLCT \\
\hline
\end{tabular}

Table 5: The in vitro Antimicrobial Activity of the Schiff base Ligand $\left(\mathrm{HL}^{1}\right)$ and its Metal(II) Complexes.

\begin{tabular}{|c|c|c|c|c|c|c|c|}
\hline Compds. & $\begin{array}{l}\text { Conc. } \\
(\mu \mathrm{g} / \mathrm{ml})\end{array}$ & S.aureus & B.subtilis & S.typhi & E.coli & A. niger & A. fumigatus \\
\hline \multirow[t]{4}{*}{$\mathrm{HL}^{1}$} & 30 & $30.00 \pm 0.00^{\mathrm{a}}$ & $32.67 \pm 0.33^{\mathrm{e}}$ & $43.00 \pm 0.00^{\mathrm{a}}$ & $20.00 \pm 0.00^{\mathrm{a}}$ & $\mathrm{R}$ & $0.00 \pm 0.00^{\mathrm{a}}$ \\
\hline & 20 & $24.67 \pm 0.33^{\mathrm{b}}$ & $26.00 \pm 0.00^{\mathrm{f}}$ & $37.33 \pm 0.33^{\mathrm{b}}$ & $14.33 \pm 0.33^{\mathrm{b}}$ & $\mathrm{R}$ & $0.00 \pm 0.00^{\mathrm{a}}$ \\
\hline & 10 & $19.67 \pm 0.33^{c}$ & $20.00 \pm 0.00^{\mathrm{g}}$ & $31.00 \pm 0.00^{c}$ & $10.000 \pm 0.00^{\mathrm{c}}$ & $\mathrm{R}$ & $0.00 \pm 0.00^{\mathrm{a}}$ \\
\hline & 30 & $40.00 \pm 0.00^{\mathrm{g}}$ & $36.33 \pm 0.33^{\mathrm{a}}$ & $50.00 \pm 0.00^{\mathrm{f}}$ & $25.00 \pm 0.00^{\mathrm{e}}$ & $\mathrm{R}$ & $20.00 \pm 0.00^{\mathrm{b}}$ \\
\hline \multirow[t]{3}{*}{$\mathrm{Ni}\left(\mathrm{HL}^{1}\right) \cdot 2 \mathrm{H}_{2} \mathrm{O}$} & 20 & $33.33 \pm 0.33^{\mathrm{d}}$ & $30.00 \pm 0.00^{\mathrm{b}}$ & $42.33 \pm 0.33^{\mathrm{a}}$ & $19.33 \pm 0.33^{\mathrm{a}}$ & $\mathrm{R}$ & $13.33 \pm 0.33^{\mathrm{c}}$ \\
\hline & 10 & $26.00 \pm 0.00^{\mathrm{b}}$ & $23.67 \pm 0.33^{\mathrm{c}}$ & $34.33 \pm 0.33^{\mathrm{d}}$ & $13.00 \pm 0.00^{\mathrm{d}}$ & $\mathrm{R}$ & $9.33 \pm 0.33^{\mathrm{d}}$ \\
\hline & 30 & $30.00 \pm 0.00^{\mathrm{a}}$ & $43.00 \pm 0.00^{\mathrm{h}}$ & $48.00 \pm 0.00^{\mathrm{g}}$ & $28.00 \pm 0.00^{f}$ & $\mathrm{R}$ & $0.00 \pm 0.00^{\mathrm{a}}$ \\
\hline \multirow[t]{3}{*}{$\mathrm{Mn}\left(\mathrm{HL}^{1}\right) \cdot 2 \mathrm{H}_{2} \mathrm{O}$} & 20 & $24.00 \pm 0.00^{\mathrm{b}}$ & $36.33 \pm 0.33^{\mathrm{a}}$ & $40.67 \pm 0.33^{\mathrm{h}}$ & $21.67 \pm 0.33^{\mathrm{g}}$ & $\mathrm{R}$ & $0.00 \pm 0.00^{\mathrm{a}}$ \\
\hline & 10 & $18.00 \pm 0.00^{\mathrm{e}}$ & $28.00 \pm 0.00^{\mathrm{i}}$ & $33.33 \pm 0.33^{\mathrm{d}}$ & $15.33 \pm 0.33^{\mathrm{b}}$ & $\mathrm{R}$ & $0.00 \pm 0.00^{\mathrm{a}}$ \\
\hline & 30 & $32.00 \pm 0.00^{\mathrm{h}}$ & $30.00 \pm 0.00^{\mathrm{b}}$ & $45.00 \pm 0.00^{\mathrm{a}^{\prime}}$ & $17.67 \pm 0.33^{\mathrm{h}}$ & $\mathrm{R}$ & $0.00 \pm 0.00^{\mathrm{a}}$ \\
\hline \multirow[t]{2}{*}{$\mathrm{Zn}\left(\mathrm{HL}^{1}\right) \cdot 2 \mathrm{H}_{2} \mathrm{O}$} & 20 & $25.00 \pm 0.00^{\mathrm{bb}}$ & $23.00 \pm 0.00^{\mathrm{c}}$ & $37.67 \pm 0.33^{\mathrm{b}}$ & $13.00 \pm 0.00^{\mathrm{d}}$ & $\mathrm{R}$ & $0.00 \pm 0.00^{\mathrm{a}}$ \\
\hline & 10 & $18.00 \pm 0.00^{\mathrm{e}}$ & $16.67 \pm 0.33^{\mathrm{d}}$ & $30.33 \pm 0.33^{\mathrm{c}}$ & $9.00 \pm 0.00^{\mathrm{c}}$ & $\mathrm{R}$ & $0.00 \pm 0.00^{\mathrm{a}}$ \\
\hline Ofloxacin & 30 & $18.00 \pm 0.00^{\mathrm{e}}$ & $16.00 \pm 0.00^{\mathrm{d}}$ & $20.00 \pm 0.00^{\mathrm{e}}$ & $15.00 \pm 0.00^{\mathrm{b}}$ & - & - \\
\hline Ketoconazole & 30 & - & - & - & - & $30.00 \pm 0.00$ & $30.00 \pm 0.00^{\mathrm{f}}$ \\
\hline
\end{tabular}


Table 6: The in vitro Antimicrobial Activity of the Schiff base Ligand $\left(\mathrm{HL}^{2}\right)$ and its Metal (II) Complexes.

\begin{tabular}{llllllll}
\hline Compds. & $\begin{array}{l}\text { Conc. } \\
(\boldsymbol{\mu g} / \mathbf{m l})\end{array}$ & S.aureus & B.subtilis & S.typhi & E.coli & A. niger & $\begin{array}{l}\text { A. } \\
\text { fumigatus }\end{array}$ \\
\hline & 30 & $29.67 \pm 0.33^{\mathrm{a}}$ & $35.00 \pm 0.33^{\mathrm{f}}$ & $37.33 \pm 0.33^{\mathrm{g}}$ & $30.00 \pm 0.00^{\mathrm{d}}$ & $\mathrm{R}$ & $\mathrm{R}$ \\
$\mathrm{HL}^{2}$ & 20 & $24.0 \pm 0.00^{\mathrm{b}}$ & $26.33 \pm 0.33^{\mathrm{g}}$ & $31.33 \pm 0.33^{\mathrm{h}}$ & $24.00 \pm 0.00^{\mathrm{e}}$ & $\mathrm{R}$ & $\mathrm{R}$ \\
& 10 & $18.67 \pm 0.33^{\mathrm{c}}$ & $20.00 \pm 0.00^{\mathrm{h}}$ & $25.00 \pm 0.00^{\mathrm{i}}$ & $18.33 \pm 0.33^{\mathrm{f}}$ & $\mathrm{R}$ & $\mathrm{R}$ \\
& 30 & $10.00 \pm 0.00^{\mathrm{e}}$ & $15.33 \pm 0.33^{\mathrm{a}}$ & $20.00 \pm 0.00^{\mathrm{a}}$ & $0.00 \pm 0.00^{\mathrm{a}}$ & $\mathrm{R}$ & $\mathrm{R}$ \\
$\mathrm{Ni}\left(\mathrm{HL}^{2}\right)_{2} 2 \mathrm{H}_{2}$ & 20 & $0.00 \pm 0.00^{\mathrm{d}}$ & $10.00 \pm 0.00^{\mathrm{b}}$ & $13.33 \pm 0.33^{\mathrm{bb}}$ & $0.00 \pm 0.00^{\mathrm{a}}$ & $\mathrm{R}$ & $\mathrm{R}$ \\
$\mathrm{O}$ & 10 & $0.00 \pm 0.00^{\mathrm{d}}$ & $0.00 \pm 0.00^{\mathrm{c}}$ & $9.00 \pm 0.00^{\mathrm{c}}$ & $0.00 \pm 0.00^{\mathrm{a}}$ & $\mathrm{R}$ & $\mathrm{R}$ \\
& 30 & $18.67 \pm 0.33^{\mathrm{c}}$ & $15.00 \pm 0.00^{\mathrm{a}}$ & $20.00 \pm 0.00^{\mathrm{a}}$ & $0.00 \pm 0.00^{\mathrm{a}}$ & $\mathrm{R}$ & $\mathrm{R}$ \\
$\mathrm{Mn}\left(\mathrm{HL}^{2}\right)_{2} .2 \mathrm{H}_{2}$ & 20 & $13.00 \pm 0.00^{\mathrm{f}}$ & $10.33 \pm 0.33^{\mathrm{b}}$ & $14.00 \pm 0.00^{\mathrm{b}}$ & $0.00 \pm 0.00^{\mathrm{a}}$ & $\mathrm{R}$ & $\mathrm{R}$ \\
$\mathrm{O}$ & 10 & $8.00 \pm 00.00^{\mathrm{g}}$ & $7.00 \pm 0.00^{\mathrm{i}}$ & $9.00 \pm 0.00^{\mathrm{c}}$ & $0.00 \pm 0.00^{\mathrm{a}}$ & $\mathrm{R}$ & $\mathrm{R}$ \\
& 30 & $30.00 \pm 0.00^{\mathrm{a}}$ & $30.00 \pm 0.00^{\mathrm{d}}$ & $35.33 \pm 0.33^{\mathrm{d}}$ & $15.33 \pm 0.33^{\mathrm{b}}$ & $\mathrm{R}$ & $\mathrm{R}$ \\
$\mathrm{Zn}\left(\mathrm{HL}^{2}\right)_{2} \cdot 2 \mathrm{H}_{2}$ & 20 & $23.67 \pm 0.33^{\mathrm{b}}$ & $24.00 \pm 0.00^{\mathrm{e}}$ & $28.67 \pm 0.33^{\mathrm{e}}$ & $10.33 \pm 0.33^{\mathrm{c}}$ & $\mathrm{R}$ & $\mathrm{R}$ \\
$\mathrm{O}$ & 10 & $18.00 \pm 0.00^{\mathrm{c}}$ & $18.67 \pm 0.33^{\mathrm{j}}$ & $21.67 \pm 0.33^{\mathrm{f}}$ & $0.00 \pm 0.00^{\mathrm{a}}$ & $\mathrm{R}$ & $\mathrm{R}$
\end{tabular}

NB. Different superscripts along the same column are significantly $(\mathrm{P}<0.05)$ different.

\section{DISCUSSION}

The Schiff bases were afforded as crystalline light yellowish derivatives of ofloxacin condensed with some amines (ophenylenediamine and 2-aminopyridine-3carboxylic acid) in a ratio $1: 2$ or $1: 1$ (amine:oflox) to obtain $\mathrm{HL}^{1}$ and $\mathrm{HL}^{2}$ Schiff bases respectively. The ligands on interaction with chlorides of $\mathrm{Ni}$ (II) and $\mathrm{Mn}(\mathrm{II})$; and $\mathrm{Zn}$ (II)Nitrate yielded complexes corresponding to the general formula [ML] and $\left[\mathrm{M}(\mathrm{L})_{2}\right]$ for $\mathrm{HL}^{1}$ and $\mathrm{HL}^{2}$ Schiff bases respectively. An appreciable percentage yield of all the new compounds were obtained which ranged from $61 \%-83 \%$. The ligands and their metal (II) complexes were observed to be stable under normal conditions with various colours characteristics of the transition metals (Aliyu and Ado, 2011) and mainly attributed to the d-d electron transition (Oladipo et al., 2005), except for zinc(II) complexes which showed yellowish and pale yellow colours which may be due to MLCT transition (Vogler and Kunkely, 2000). The complexes showed a steady trend of higher melting points than those of the ligands which may be due to the inter-molecular bonding as a result of metallic lattice and increase in molecular weight (Ogunniran et al., 2008). The melting points of the ligands and their complexes range from $134{ }^{\circ} \mathrm{C}\left(\mathrm{HL}^{2}\right)-189{ }^{\circ} \mathrm{C}$ $\mathrm{Zn}-\left(\mathrm{HL}^{2}\right)_{2} \cdot 2 \mathrm{H}_{2} \mathrm{O}$. The consistent range difference of $+2{ }^{\circ} \mathrm{C}$ melting points observed indicates that the synthesized compounds are presumed pure Table 1. The structures of the Schiff base ligands and their metal(II) complexes suggested from the theoretical elemental analysis and metal estimates agree 
to some extent with their proposed formulae (Sadeek et al., 2014). The molar conductance measurements of the ligands and the complexes in $10^{-3} \mathrm{M}$ DMSO range from 0.053 - $0.70 \mathrm{Scm}^{2} \mathrm{~mol}^{-1}$ for $\mathrm{HL}^{1}$ and $\mathrm{Mn}$ $\left(\mathrm{HL}^{2}\right)_{2} \cdot 2 \mathrm{H}_{2} \mathrm{O}$ complex which are relatively low, indicating that the new compounds are non-electrolytes in nature (Ndahi and Nasiru, 2012).

\section{FT-Infrared}

The IR absorption spectra of the ofloxacin drug, amines used and, Schiff base ligands $\left(\mathrm{HL}^{1} \& \mathrm{HL}^{2}\right)$ and their complexes with Ni (II), Mn (II) and Zn (II) are presented in Table 3. The infrared spectra of fluoroquinolone compounds are observed to be complex as a result of several functional moieties in their structures and hence, only two linkage sites are feasible in condensation and coordination (George, 2008). The most common vibrations considered as important region in the ofloxacin IR spectra appeared at 1519 and $2924 \mathrm{~cm}^{-1}$ for $\mathrm{v}(\mathrm{C}=\mathrm{N})$ and $\mathrm{v}\left(\mathrm{NH}_{2}\right)$ vibrations respectively. The spectra of the two amines, o-phenylenediamine (o-phdn) and 2aminopyridine-3-carboxylic acid (2-Apdn) showed $\mathrm{v}(\mathrm{C}=\mathrm{N})$ vibration bands at higher values than the ligands at 1627 and $1697 \mathrm{~cm}^{-1}$ respectively as against 1620 and $1631 \mathrm{~cm}^{-1}$ in $\mathrm{HL}^{1}$ and $\mathrm{HL}^{2}$ ligands spectra. The amines also showed a distinctive $\mathrm{V}\left(\mathrm{NH}_{2} / \mathrm{N}-\mathrm{H}\right)$ vibration band at 3363 and $3255 \mathrm{~cm}^{-1}$ respectively which is absent in the ligands and their metal complexes due to coordination (Patel et al., 2011a). The Schiff base ligands $\left(\mathrm{HL}^{1} \& \mathrm{HL}^{2}\right)$ showed a typical characteristic azomethine $\mathrm{v}(\mathrm{C}=\mathrm{N})$ vibration band at 1620 and $1631 \mathrm{~cm}^{-1}$ and the absence of $\mathrm{v}(\mathrm{N}-\mathrm{H})$ vibrations of the amines between $2785-3363 \mathrm{~cm}^{-1}$ in the ligands further established the condensation of the drug with the amines which afforded the two ligands $\left(\mathrm{HL}^{1} \& \mathrm{HL}^{2}\right)$.

On complexation with metal(II), the $\mathrm{v}(\mathrm{C}=\mathrm{N})$ band shifted to higher frequency (Nakamoto, 2006) at the following regions; 1643 and $1639 \mathrm{~cm}^{-1}$ [Ni(II)], 1631 and 1639 $\mathrm{cm}^{-1}[\mathrm{Mn}(\mathrm{II})], 1635$ and $1635 \mathrm{~cm}^{-1}$ [Zn(II)] complexes respectively (Table 3 ). The shift from the azomethine stretching vibrations of the ligands observed in the spectral bands of the complexes may be ascribed to the coordination of the azomethine nitrogen to the metal (II) ions (Reddy et al., 2008). Also, the IR spectra of the drug and the amine, ofloxacin, o-phenylenediamine (o-phdn) and 2-aminopyridine (2-Apdn) showed the $\mathrm{v}(\mathrm{C}-\mathrm{N})$ stretching vibration band at 1458, 1496 and $1458 \mathrm{~cm}^{-1}$ respectively. The o-phdn IR presented this band at higher frequency of $1496 \mathrm{~cm}^{-1}$ than the $\mathrm{HL}^{1}$ and its metal complexes which exhibited this spectral band at a range of $1462-1489 \mathrm{~cm}^{-1}$. The $\mathrm{HL}^{2}$ and its metal complexes displayed the $\mathrm{v}(\mathrm{C}-\mathrm{N})$ vibration band at a higher range of 1419-1481 $\mathrm{cm}^{-1}$ than its amine (2-Apdn) which is displayed at $1458 \mathrm{~cm}^{-1}$.

The IR spectra of the complexes displayed a discrete non ligand band with low intensity at $516-682 \mathrm{~cm}^{-1}$ for $\mathrm{HL}^{1}$-complexes and 578-663 $\mathrm{cm}^{-1}$ for $\mathrm{HL}^{2}$-complexes corresponding to $\mathrm{v}(\mathrm{M}-\mathrm{N})$ stretching vibrations. The band at $405-613 \mathrm{~cm}^{-1}$ for $\mathrm{HL}^{1}$ complexes and at $405-478 \mathrm{~cm}^{-1}$ for $\mathrm{HL}^{2}-$ complexes is attributed to $\mathrm{v}(\mathrm{M}-\mathrm{O})$ stretching vibration mode which are similar to the reported works of Sultana et al. (2013); Verma et al. (2013); Patel et al. (2012); Ndahi et al. (2012) and Imran et al. (2007). These non-ligand spectral bands are indicators to the possible coordination of the azomethine nitrogen and the carboxylato oxygen to the metal ions (Sadeek et al., 2005). A broad diffuse bands of medium and sharp intensity in the regions $\left(3255-3406 \mathrm{~cm}^{-1}\right)$ is assigned to the $\mathrm{OH}$ stretching vibrations of the $\mathrm{COOH}$ group of Ofloxacin and 2-amino- pyridine-3carboxylic acid in both the Schiff base ligands and the complexes. Similarly, the weak and medium bands appearing at 790-995 $\mathrm{cm}^{-1}$ corresponded with the stretching vibration of the product water molecules (Anacona and Toledo, 2001). However, worth noting also is the absence of a pair of band expected at 3245 and $3309 \mathrm{~cm}^{-1}$ corresponding to $\mathrm{v}\left(\mathrm{NH}_{2}\right)$ of the amine moieties used which further confirmed the coordination of the drug Ofloxacin to the two amines forming azomethine linkage (Patel et al., 2011b). 


\section{Electronic Spectra of the Schiff Base Ligands and their Metal Complexes}

The electronic spectral band of the free ligands and the metal complexes studied in methanol (Table 5) revealed that there is $n \rightarrow \pi^{*}$ transition of the ethylenic double bond or nonbonding electrons on nitrogen atom of the azomethine bond at $32468 \mathrm{~cm}^{-1}$ and 27624 $\mathrm{cm}^{-1}$ for $\mathrm{HL}^{1}$ and $\mathrm{HL}^{2}$ ligands respectively assigned to $\mathrm{v}(\mathrm{C}=\mathrm{O}), \mathrm{v}(\mathrm{C}=\mathrm{N})$ and $\mathrm{v}(\mathrm{OH})$ vibration groups (Nagajothi et al., 2013). In the metal complexes, only broad bands were seen in the visible region which are probably due to d-d transition and MLCT (Ogunniran et al., 2008).

The Ni(II) complexes exhibited electronic spectral band in the UV at higher intensities than the free ligands at 33898 and $42017 \mathrm{~cm}^{-1}$ for $\mathrm{Ni}\left(\mathrm{HL}^{1}\right) \cdot 2 \mathrm{H}_{2} \mathrm{O}$ and $\mathrm{Ni}\left(\mathrm{HL}^{2}\right)_{2} \cdot 2 \mathrm{H}_{2} \mathrm{O}$ complexes respectively attributed to $n \rightarrow \pi^{*}$ and $\pi \rightarrow \pi^{*}$ transitions of the non-bonding and Pi electrons (Patel et al., 2012). These transitions occur in case of unsaturated hydrocarbons which contain ketone or azomethine group (Chohan, 2001; Sadeek et al., 2014). Mn(II) complexes showed bands at $29155 \mathrm{~cm}^{-1}$ and $45249 \mathrm{~cm}^{-1}$ respectively, which may be assigned to MLCT and ${ }^{6} \mathrm{~A}_{1 \mathrm{~g}} \rightarrow{ }^{4} \mathrm{~T}_{2 \mathrm{~g}}$ or $\pi \rightarrow \pi^{*}$ transitios (Ndahi et al., 2012; Sadeek and EL-Shwiniy, 2010). The electronic spectral bands for $\mathrm{Zn}$ (II) complexes were exhibited at $28653 \mathrm{~cm}^{-1}$ and $28329 \mathrm{~cm}^{-1}$ respectively which corresponded to MLCT transitions. However, these electronic absorption spectra results couldn't provide adequate information as determine the geometry of the novel compounds because absorptions were not observed in the visible region above $400 \mathrm{~nm}$ in the UV which may be due to other factors unchecked.

Based on the data obtained from the physicochemical studies, infrared spectral analysis and UV/Visible electronic absorption spectra, proposed structures were elucidated for $\mathrm{HL}^{1}$ and $\mathrm{HL}^{2}$ Schiff base ligands and their metal (II) complexes as shown in Figures 1-4.

\section{Ligand $\left(\mathrm{HL}^{\mathbf{1}}\right)$ and its metal complexes}

Table 5: presented the antimicrobial activity of $\mathrm{HL}^{1}$ Schiff base and its metal complexes. The derivatives synthesized showed a good to excellent activity towards all the mentioned panel of bacterial strains across the range of the serial concentrations $(30 \mu \mathrm{g} / \mathrm{ml}, 20 \mu \mathrm{g} / \mathrm{ml}$ and $10 \mu \mathrm{g} / \mathrm{ml}$ ) compared with the parent drug ofloxacin and most were significant $(\mathrm{P}<0.05)$ on one way ANOVA analysis. On the fungal strains bioassay, moderate activity was observed for $\mathrm{Ni}\left(\mathrm{HL}^{1}\right) \cdot 2 \mathrm{H}_{2} \mathrm{O}$ complex in A. fumigatus strain compared to the reference drug ketoconazole; while the rest of the new compounds showed no activity. However, A. niger strain of the fungi didn't show activity in all the tested compounds. The increasing activity of the metal complexes against the free ligands $\mathrm{HL}^{1}$ may be explained on the basis of oxidation state, overtone concept and chelation theory (Osowole et al., 2008) which reduces polarity of the metal ion by partial sharing of the positive charge with donor atoms of the ligand (imine \& oxygen). This atomic shake-up increases the lipophilic character, favouring the permeation through lipid layers of the bacterial membrane and consequently restricting further growth of the organism (Obalaye et al., 2011). However, distinctively, $\mathrm{HL}^{1}$ free ligand showed good activity on $S$. typhi at all dilutions (Table 5), followed by $B$. subtilis, S. aureus and least on E. coli $(43.00 \pm 0.00, \quad 32.67 \pm 0.33, \quad 30.00 \pm 0.00$ and $20.00 \pm 0.00) \mathrm{mm}$ respectively compared to the parent drug ofloxacin values. No activity was observed in the fungal strains (A. niger and $A$. fumigatus) by the free ligand $\mathrm{HL}^{1}$. This potency of the $\mathrm{HL}^{1}$ ligand was sustained by its metal complexes in which $\mathrm{Ni}\left(\mathrm{HL}^{1}\right) \cdot 2 \mathrm{H}_{2} \mathrm{O}$ exhibited excellent activity on $S$. typhi $(50.00 \pm 0.00,42.33 \pm 0.33$ and $34.33 \pm 0.33) \mathrm{mm}$ inhibitions at $30, \quad 20 \quad \& \quad 10 \mu \mathrm{g} / \mathrm{ml}$ concentrations compared to $20.00 \pm 0.00 \mathrm{~mm}$ at $30 \mu \mathrm{g} / \mathrm{ml}$ for parent drug (ofloxacin). Very good activity were also observed in $S$. aureus $(40.00 \pm 0.00,33.33 \pm 0.33$ and $26.00 \pm 0.00) \mathrm{mm}$ B. subtilis $(36.33 \pm 0.33, \quad 30.00 \pm 0.00$ and $23.63 \pm 0.33) \mathrm{mm}$ and E. coli $(25.00 \pm 0.00$, $19.33 \pm 0.00$ and $13.00 \pm 0.00) \mathrm{mm}$ compared with the parent drug ofloxacin with $(18.00 \pm 0.00,16.00 \pm 0.00$ and $15.00 \pm 0.00) \mathrm{mm}$ respectively. The activity of the ligand and its 
complexes on the test organisms followed the trend $\mathrm{Ni}>\mathrm{Mn}>\mathrm{Zn}>\mathrm{HL}^{1}$ and these variations were tested to be significantly $(\mathrm{P}<0.05)$ different on ANOVA statistical tool.

\section{Ligand $\left(\mathrm{HL}^{2}\right)$ and its metal complexes}

The preliminary antibacterial screening results for $\mathrm{HL}^{2}$ and its metal complexes are presented in Table 6 . These set of ofloxacin derivatives presented a divergent activity from the common and popular higher activity by the complexes against the free ligands. In this study, the free ligand $\mathrm{HL}^{2}$ exhibited higher and good in vitro activity than the complexes in the entire representative bacterial strains used with the highest inhibition diameter measured in S. typhi followed by B. subtilis, E. coli and $S$. aureus, showing more potency on Gram negative at all levels of the concentrations. The $\mathrm{HL}^{2}$ metal complexes showed moderate and good activity on some of the test organisms. However, to the contrary from the previous experience with $\mathrm{HL}^{1}$ ligand and its complexes, the activity of the $\mathrm{M}\left(\mathrm{HL}^{2}\right)_{2} \cdot 2 \mathrm{H}_{2} \mathrm{O}$ complexes appeared to be lower than the values observed for the ligand; with the $\mathrm{Zn}\left(\mathrm{HL}^{2}\right)_{2} \cdot 2 \mathrm{H}_{2} \mathrm{O}$ complex showing higher activity in all the probed bacterial strains with the highest inhibition zone marked in S. typhi followed by B. subtilis, $S$. aureus and $E$. coli with inhibitory diameter of $35.33 \pm 0.33, \quad 30.00 \pm 0.00, \quad 30.00 \pm 0.00$ and $15.33 \pm 0.33 \mathrm{~mm}$ (SEM) respectively at 30 $\mu \mathrm{g} / \mathrm{ml}$ dilution (Table 6). Ni $\left(\mathrm{HL}^{2}\right)_{2} \cdot 2 \mathrm{H}_{2} \mathrm{O}$ complexes showed activity only at $30 \mu \mathrm{g}$ dilution in $S$. aureus and no activity at 10 $\mu \mathrm{g} / \mathrm{ml}$ in $B$. subtilis. This primary investigation also revealed that $\mathrm{Ni}$ $\left(\mathrm{HL}^{2}\right)_{2} .2 \mathrm{H}_{2} \mathrm{O}$ and $\mathrm{Mn}\left(\mathrm{HL}^{2}\right)_{2} \cdot 2 \mathrm{H}_{2} \mathrm{O}$ did not show activity in E. coli. In cases where activity were observed, the extent of the in vitro inhibitions range from moderate to good potency compared to the parent drug. Furthermore, the antimicrobial assay of the $\mathrm{HL}^{2}$ ligand and the complexes revealed no activity in the two fungal strains (A. niger and A. fumigatus) used.

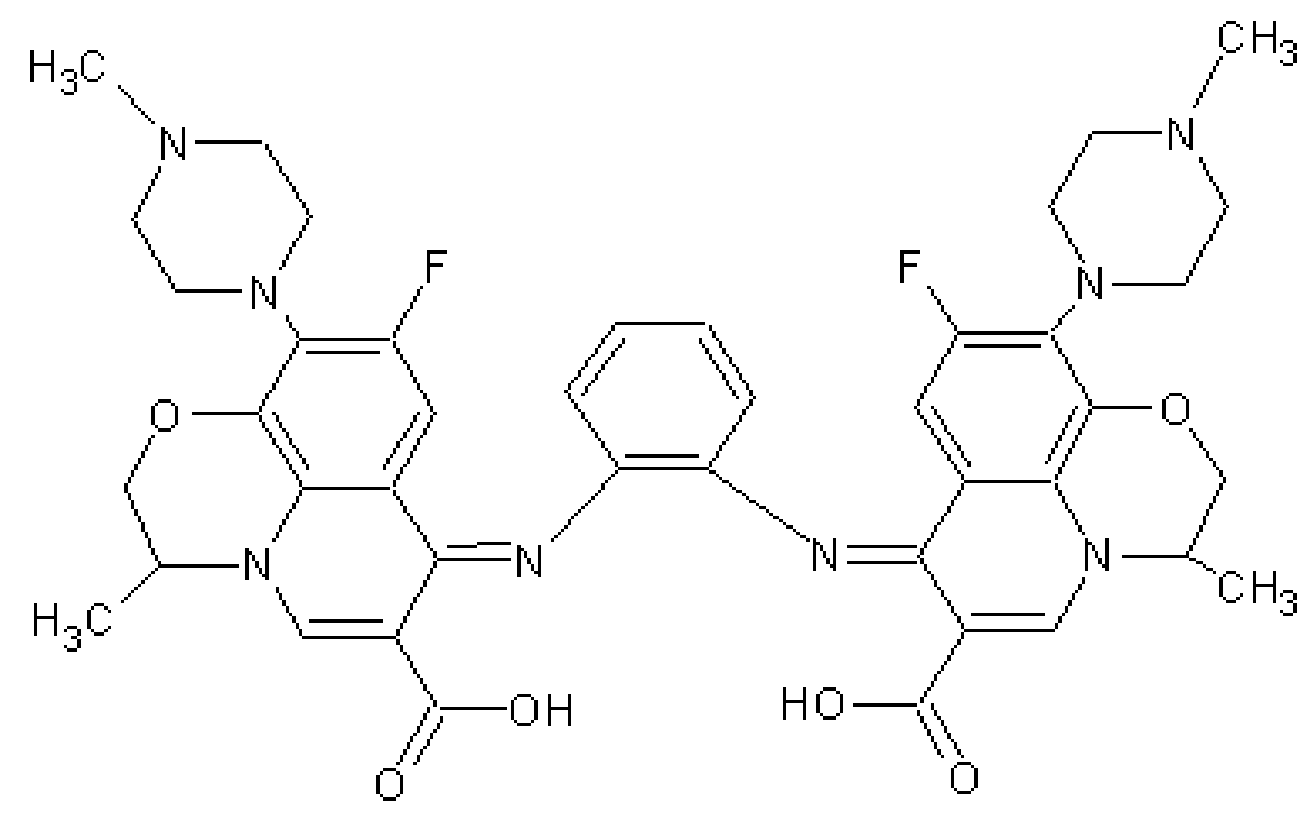

Figure 1: Proposed Structure for Ofloxo-o-phenylenediamine Ligand $\left(\mathrm{HL}^{1}\right)$. 


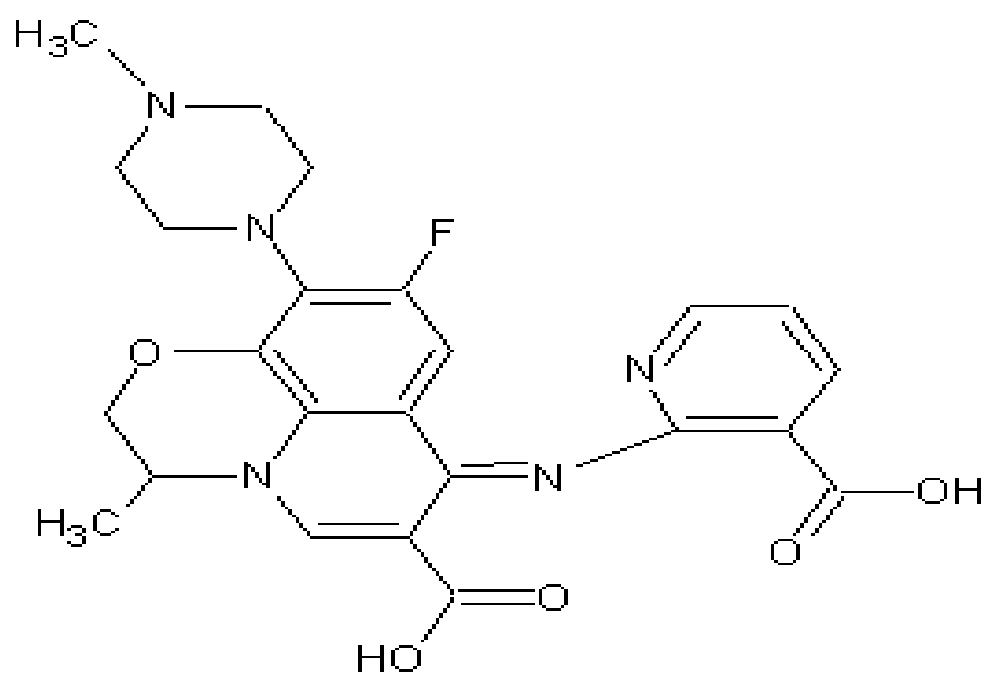

Figure 2: Proposed Structure for Ofloxo-2-Apydn Ligand $\left(\mathrm{HL}^{2}\right)$

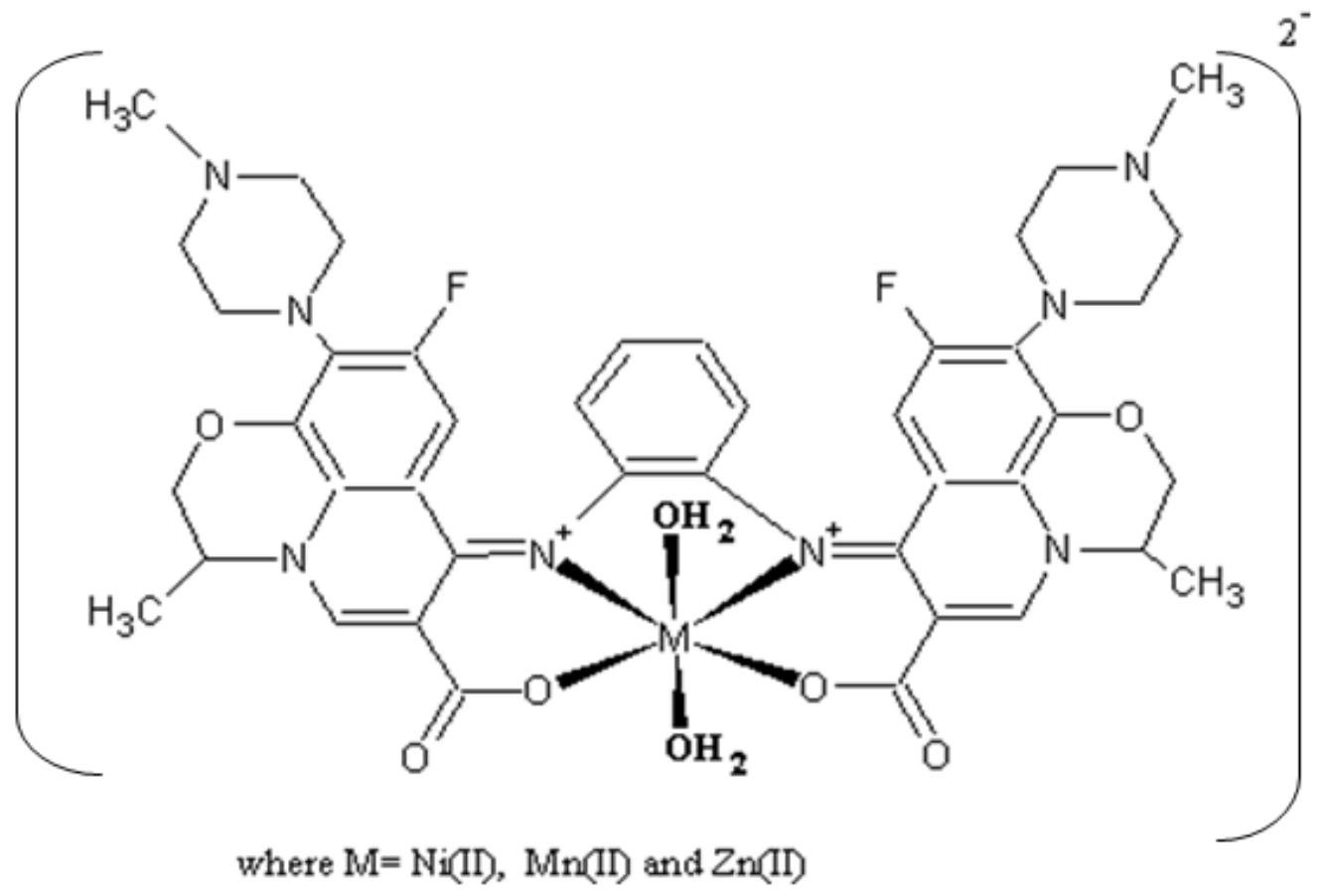

Figure 3: Proposed Structure for $\mathrm{HL}^{1}$ Metal Complexes. 


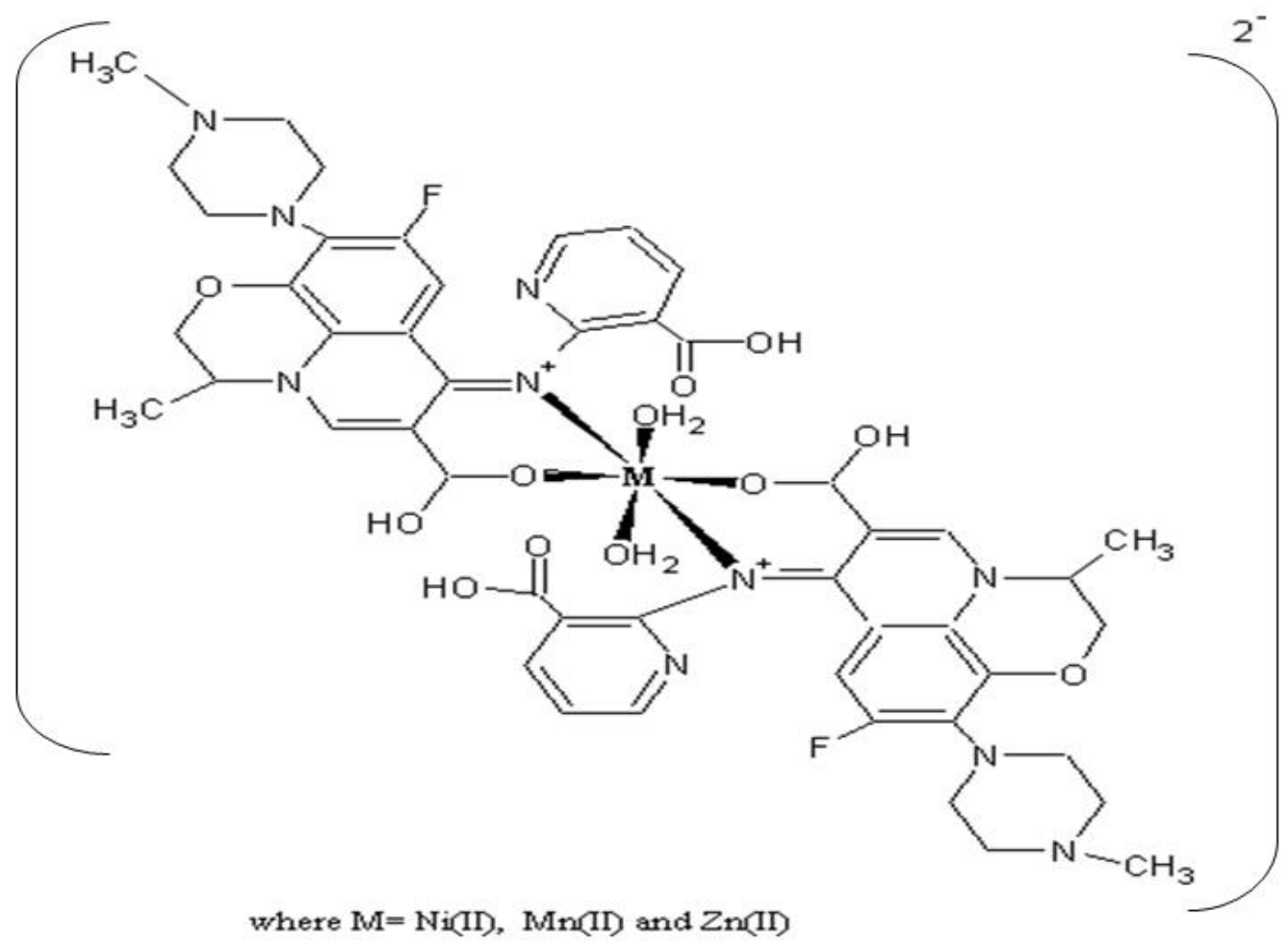

Figure 4: Proposed Structure for $\mathrm{HL}^{2}$ Metal Complexes.

\section{Conclusion}

In continuous search for better activity drugs ahead of the existing antimicrobial agents which have been severally reported to show a declining action against todays' pathogens due to many human and behavioural factors; this work was designed to obtain or otherwise an improved antimicrobial agents through modification of the existing drugs (ofloxacin); using some Schiff bases containing ofloxacin drug with amines. These ligands were complexed with some transition metals and the newly synthesized compounds characterized and their antimicrobial potency evaluated. At the end of the analysis, the work afforded stable ofloxacin derivatives from Schiff bases derived from ophenylenediamine and 2-aminopyridine-3carboxylic acid and their metal complexes. The characterization results suggested 2:1 and 1:1 mole ratio for ofloxacin:amine condensation while $1: 1$ and $1: 2$ ratio was found for metal:ligand combinations. The novel compounds presented promising to excellent antimicrobial activity on the tested panel of microbes which is a positive breakthrough toward providing a lead to attainment of more improved antimicrobial agents.

\section{COMPETING INTERESTS}

The authors declare that they have no competing interests

\section{AUTHORS' CONTRIBUTIONS}

HANP designed, collected, processed data and wrote the article; NPN was instrumental in the conception and design of the research and supervised the whole work to its success; HSB supervised the antimicrobial aspect of the research; GM contributed to the design, participated in analysis of the noble products obtained and immensely facilitated the publication in this reputable journal; AAO read the manuscript several times and made relevant inputs as Co-Supervisor; and HG was 
active part of the laboratory works and also participated in article writing.

\section{ACKNOWLEDGEMENTS}

The authors acknowledge Tertiary Education Trust Fund (TET-FUND) AbujaNigeria and Management of Ramat Polytechnic Maiduguri, Borno State Nigeria for sponsoring the research. We also appreciated the contributions of Chemistry Department and Veterinary Microbiology Department Laboratories of University of Maiduguri, Borno State Nigeria where all the data were generated and analyzed.

\section{REFERENCES}

Al-Bayati RIH, Mahdi FR, Ahmed AHA. 2010. Synthesis, Spectroscopic and Antimicrobial Studies of Transition Metal Complexes of $\mathrm{N}$-amino Quinolone Derivatives. $14^{\text {th }}$ International Electronic Conference of Synthetic Organic Chemistry, (ELSOC-14): pp. 1-30.

Aliyu HN, Ado I. 2010. Studies of Mn (II) and Ni(II) Complexes with Schiff base derived from 2-amino benzoic acid and Salicylaldehyde. Bayero J. Pure Appl. Sci., 3(1): 9-16. $\quad$ DOI: https://doi.org/10.4314/bajopas.v3i1.588 03

Akpa SJ, Zoakouma RSP, Say VM, Ouattara M, Kone MW, Sissouma D, Adjou A. 2016. Synthesis and in vitro antifungal evaluation of 2-thioalkylarylbenzimidazoles derivatives against Candida albicans. Int. J. Biol. Chem. Sci., 10(3): 1403-1412. DOI: https://dx.doi.org/10.4314/ijbcs.v10i3.38

Anacona JR, Toledo C. 2001. Synthesis and Antibacterial Activity of Metal Complexes of Ciprofloxacin. J. Transit. Met. Chem., 26(1): 228-231. DOI: https://doi.org/10.1023/a:100715481708 1

Anand SP, Doss A, Nandagopalan V. 2011. Antibacterial Studies of Clitoria ternatea Linn. A high Potential Medicinal Plant. Int. J. Appl. Biol. Pharm. Tech.,2: 453456.

DOI: http://www.ijabpt.com/pdf/15063Doss\% 20andro\%5B1\%5D.pdf.
Chohan ZH. 2001. Synthesis, Characterization and Biological Properties of Bivalent Transition Metal Complexes of Co (II), $\mathrm{Cu}$ (II), Ni (II) and $\mathrm{Zn}$ (II) with some Acylhydrazine Derived from Furanyl and Thienyl ONO and SNO donor Schiff base Ligands. Synth. React. Inorg. MetOrg. Chem., 31(1): 1-16. DOI: https://doi.org/10.1081/sim-100001928

Gangani BJ, Parsania PH. 2014. Ultrasonic Speed and Related Acoustical Parameters of Symmetric Double Schiff Bases Solutions at 308.15K. J. Chem. Pharm. Res., 6(11): 243-247.

George P. 2008. Mononuclear Metal Complexes with Ciprofloxacin: Synthesis, Characterization and DNABinding Properties. J. Inorg. Biochem., 102(12): 1798-1811. DOI: https://doi.org/10.1016/j.jinorgbio.2008. 05.012

Imran M, Igbal J, Iqbal S, Ijaz N. 2007. In vitro Antibacterial Studies of Ciprofloxacin-imines and their Complexes with $\mathrm{Cu}$ (II), $\mathrm{Ni}$ (II) $\mathrm{Co}$ (II) and Zn (II). Turk. J. Biol., 31(2): 67-72. DOI:

http://journals.tubitak.gov.tr/biology/issu es/biy-07-31-2/biy-31-2-2-0610

Jayashree BS, Kaur M, Pai A. 2012. Synthesis, Characterization, Antioxidant and Anticancer Evaluation of Novel Schiff bases of 2-quinolones. Elixier Org. Chem., 52: 11317-11322. DOI: http://www.elixirpublishers.com/articles/ 1352272748_52\%20\%282012\%29\%201 1317-11322.pdf

Nagajothi A, Kiruthika A, Chitra S, Parameswari K. 2013. Fe (III) Complexes with Schiff base Ligands: Synthesis, Characterization and Antimicrobial Studies. Res. J. Chem. Sci., 3(2): 35-43. DOI: https://scholar.google.com/scholar?hl

Nakamoto K. 2006. "Infrared and Raman Spectra of Inorganic and Coordination Compounds: Handbook of Vibrational Spectroscopy, 2006 - Wiley Online Library Print ISBN: 9780471988472| Online 
ISBN: $9780470027325 \mid$ DOI: $10.1002 / 0$ 470027320.

Ndahi NP, Nasiru YP. 2012. Complexes of Cobalt (II), Nickel (II) and Zinc (II) with Schiff bases Derived from 4anisaldehyde. Int. J. Pharm. Sci. Res., 3(12): 5116-5120. DOI: http://dx.doi.org/10.13040/IJPSR.09758232.3(12).5116-20

Ndahi NP, Pindiga YN, Sandabe UK. 2012. Synthesis, Characterization and Antibacterial Studies of some Schiff base Complexes of Cobalt (II), Nickel (II) and Zinc (II). Asian J. Biochem. Pharm. Res., 1(2):

2012. https://scholar.google.com/scholar?lookup Ngassam RFT, Tantse M, Kwetche PRF, Noumi DPN, Kouamouo J, Louodom JS, Kweyang BPT, Kourouma K. 2017. Multicenter study on antibiotic susceptibility/resistance trends in the western region of Cameroon. Int. J. Biol. Chem. Sci., 11(1): 131-143. DOI: http//dx.doi.org/10.4314/ijbcs.v11i1.11

Obalaye JA, Adediji JF, Adebayo MA. 2011. Synthesis and Biological Activities of Metal Complexes of 2, 5-diamino-1,3,4thiadiazole Derived from Semicarbazide Hydrochloride. Molecules., 16(7): 58615874.

DOI: https://doi.org/10.3390/molecules160758 61

Ogunniran KO, Ajanaku KO, James OO, Ajani OO, Nwinyi CO, Allensela MA. 2008. Fe (III) and Co (II) Complexes of Mixed Antibiotics: Synthesis, Characterization Antimicrobial Potential and their effect on Alkaline Phosphatase Activities of Selected Rat Tissues. Int. J. Physic. Sci., 3(8): 177182.

$\mathrm{http}: / /$ eprints.covenantuniversity.edu.ng/ 219/

Oladipo MA, Woods JAO, Odunola OA. 2005. Synthesis, Vibrational Spectra and Magnetic Properties of Cobalt (II), Nickel (II) and Copper (II) Complexes of Barbituric Acid. Sci. Foc., 10(1): 49-52. https://scholar.google.com/scholar?hl

Osowole AA, Kolawole GA, Fagade OJ. 2008. Synthesis, Characterization and
Biological Studies on Unsymmetrical Schiff base Complexes of Nickel(II), Copper(II) and Zinc(II) and Adducts with 2,2'- dipyridine and 1, 10 phenanthroline. J. Coord. Chem., 61: 1046-1049. DOI: https://doi.org/10.1080/00958970701482 446

Patel KS, Patel JC, Dholariya HR, Patel VK, Patel KD. 2012. Synthesis of $\mathrm{Cu}(\mathrm{II})$, $\mathrm{Ni}(\mathrm{II}), \mathrm{Co}(\mathrm{II})$, and $\mathrm{Mn}(\mathrm{II})$ Complexes with Ciprofloxacin and their Evaluation of Antimicrobial, Antioxidant and Antituberclular Activity; Open J. Met. Sci. Res., 2: 49-59. DOI: https://doi.org/10.4236/ojmetal.2012.230 08

Patel NB, Agravate SN, Shaikh FM. 2011a. Synthesis and Antimicrobial Activity of New Pyridine Derivatives. J. Med. Chem. Res., 20(7): 1033-1041. DOI: https://doi.org/10.1007/s00044-0109440-0

Patel VK, Patel PR, Nagar AA, Chugh NN, Bendale AR. 2011b. Sonochemical Synthesis and Antibacterial Screening of Ciproflaxacin-imines Metal ion Complexes. Asian J. Pharm. Sci. Res., 1(2): 91-99. DOI: https://www.researchgate.net/profile/Dr_ Nagar2/publication/215485196

Raman N, Kulandaisamy A, Thangaraja C. 2003. Redox and antimicrobial studies of transition metal (II) tetradendate Schiff base complexes. J. Trans. Met. Chem., 28: 29-36.

Reddy V, Patil N, Angadi SD. 2008. Synthesis, Characterization and Antimicrobial Activity of $\mathrm{Cu}$ (II), Co (II) and $\mathrm{Ni}$ (II) Complexes with $\mathrm{O}, \mathrm{N}$ and $\mathrm{S}$ donor Ligands. Europ. J. Chem., 5(3): 577-583. https://doi.org/10.1155/2008/170631

Sadeek AS, Mohammed SE, Sherif MA. 2014. Spectroscopic Investigation of New Schiff base Metal Complexes. Int. J. Advan. Res., 2(7): 90-107. http://journalijar.com/uploads/802_IJAR -3239.pdf.

Sadeek SA, EL-Shwiniy WH. 2010. Metal Complexes of the Third generation 
Quinolone Antibacterial Drug Sparfloxacin: Preparation, Structure and Microbial Evaluation. J. Coord. Chem., 63(19): $3471-3482 . \quad$ DOI: https://doi.org/10.1080/00958972.2010.5 14049

Sadeek SA, Teleb SM, Refat MS, Elmosallamy MAF. 2005. Preparation, Thermal and Vibrational Studies of $\mathrm{UO}_{2}$ (acac-o-phdn) (L) ( $\mathrm{L}=\mathrm{H}_{2} \mathrm{O}, \mathrm{Py}, \mathrm{DMF}$ and $\left.\mathrm{Et}_{3} \mathrm{~N}\right)$. J. Coord. Chem., 58(12): 1077 1085.

DOI: https://doi.org/10.1080/00958970500122 79

Schiff H. 1864. Mittheilungen aus dem Universitätslaboratorium in Pisa: Eine neue $119 . \quad$ DOI: https://doi.org/10.1002/jlac.1864131011 3 Shrikrishna DT, Dinesh VB, Satish AD, Rajendra PP. 2012. Facile and Efficient Method for Preparation of Schiff bases Catalyzed by $\mathrm{Ni}$ $\left(\mathrm{NO}_{3}\right)_{2} 6 \mathrm{H}_{2} \mathrm{O}$ under room Temperature. Int. J. Chem. Sci., 10(4):1837-1843. https://scholar.google.com/scholar?looku $\mathrm{p}$

Sridha SK, Saravan M, Ramesh A. 2001. Synthesis and antibacterial screening of hydrazones Schiff and Mannich bases of isatin derivatives. Eur. J. Med. Chem., 36: 615-623.

Sultana N, Arayne MS, Rizvi SBS, Haroon U, Mesaik MA. 2013. Synthesis,
Spectroscopic and Biological Evaluation of some Levofloxacin Metal Complexes. J. Med. Chem., 22(3): 1371-1377. DOI: https://doi.org/10.1007/s00044-0120132-9

Usman H, Osuji JC. 2008. Phytochemical and in vitro Antimicrobial Assay of the Leaf Extract of Newbouldia Laevis. Afri. J. Trad. Compl. Alt. Med., 4(4): 476-480. DOI: https://doi.org/10.4314/ajtcam.v4i4.31240 Verma S, Sirbaiya A, Pandeya S. 2013. Antimicrobial Activity of Schiff base of Ofloxacin. Asian Journal of Pharmaceutical Research and Development, 1(2): $\quad$ 56-64.

DOI:

http://www.ajprd.com/index.php/journal/ article/view/26

Vogler A, Kunkely H. 2000. Photochemistry Induced by Metal-to-Ligand Charge Transfer excitation. Coord. Chem. Rev., 208(1): $\quad 321-329 . \quad$ DOI: https://doi.org/10.1016/s00108545(99)00246-5

Waziri I, Ndahi NP, Paul BB. 2013. Synthesis, Physicochemical and Antimicrobial Studies of Co (II), Zn (II) and $\mathrm{Fe}$ (III) Mixed Antibiotics Metal Complexes. J. Chem. Pharm. Res., 5(9): 84-89. https://scholar.google.com/scholar. 